D.T. Wickramasinghe, L. Ferrario, and G.V. Bicknell, eds.

\title{
Outflow Angles and Bulk Lorentz Factors for Different Categories of AGN
}

\author{
Erick. J. Guerra, Ruth A. Daly \\ Department of Physics, Princeton University, Princeton NJ, 08544
}

\begin{abstract}
Relativistic outflows from AGN can be parameterized by $\theta$, the angle subtended by the direction of the outflow and the line of sight to the observer, and $\gamma$, the bulk Lorentz factor of the outflow. The Doppler factor, $\delta$, and the apparent speed in the plane of the sky, $\beta_{a p p}$, are combinations of $\theta$ and $\gamma$. The Doppler factor can be estimated using either the equipartition Doppler factor, $\delta_{e q}$ (Readhead 1994), or the inverse Compton Doppler factor, $\delta_{I C}$. These Doppler factor estimates are combined with observed $\beta_{a p p}$ to solve for $\theta$ and $\gamma$ for different categories of AGN.

Ghisellini et al. (1993) compute $\delta_{I C}$ for 105 compact radio sources, and Güijosa \& Daly (1996) compute $\delta_{e q}$ for the same sample. Daly, Guerra, \& Güijosa (1996) estimate $\theta$ and $\gamma$ for the 43 sources that have $\beta_{a p p}$ listed by Vermeulen \& Cohen (1994) and $\delta_{e q}$ computed by Güijosa \& Daly (1996).

Solutions and errors for $\theta$ and $\gamma$ are presented in Figures 1 and 2 using $\delta_{e q}$ and $\delta_{I C}$ respectively. Guerra \& Daly (1996) discuss these estimates and errors in greater detail. These AGN fall into the following categories: BL Lacertae objects (BL Lacs), core-dominated high-polarization quasars (CDHPQ), core-dominated low-polarization quasars (CDLPQ), core-dominated quasars with no polarization information (CDQ(NPI)), lobe-dominated quasars (LDQ), and radio galaxies ( $R G)$.
\end{abstract}

Acknowledgments. This work supported in part by the U.S. National Science Foundation through a Graduate Student Fellowhsip and a National Young Investigator Award.

\section{References}

Daly, R. A., Guerra, E. J., \& Güijosa, A. 1996, in "Energy Transport in Radio Galaxies and Quasars", eds. P. Hardee, A. Bridle, \& J. Zensus (San Francisco: ASP conf. series), 73

Ghisellini, G., Padovani, P., Celotti, A., \& Maraschi, L. 1993, ApJ, 407, 65

Guerra, E. J. \& Daly, R. A. 1996, ApJ, submitted

Güijosa, A. \& Daly, R. A. 1996, ApJ, 461, 600

Readhead, A. C. S. 1994, ApJ, 426, 51

Vermeulen, R. C. \& Cohen, M. H. 1994, ApJ, 430, 467 


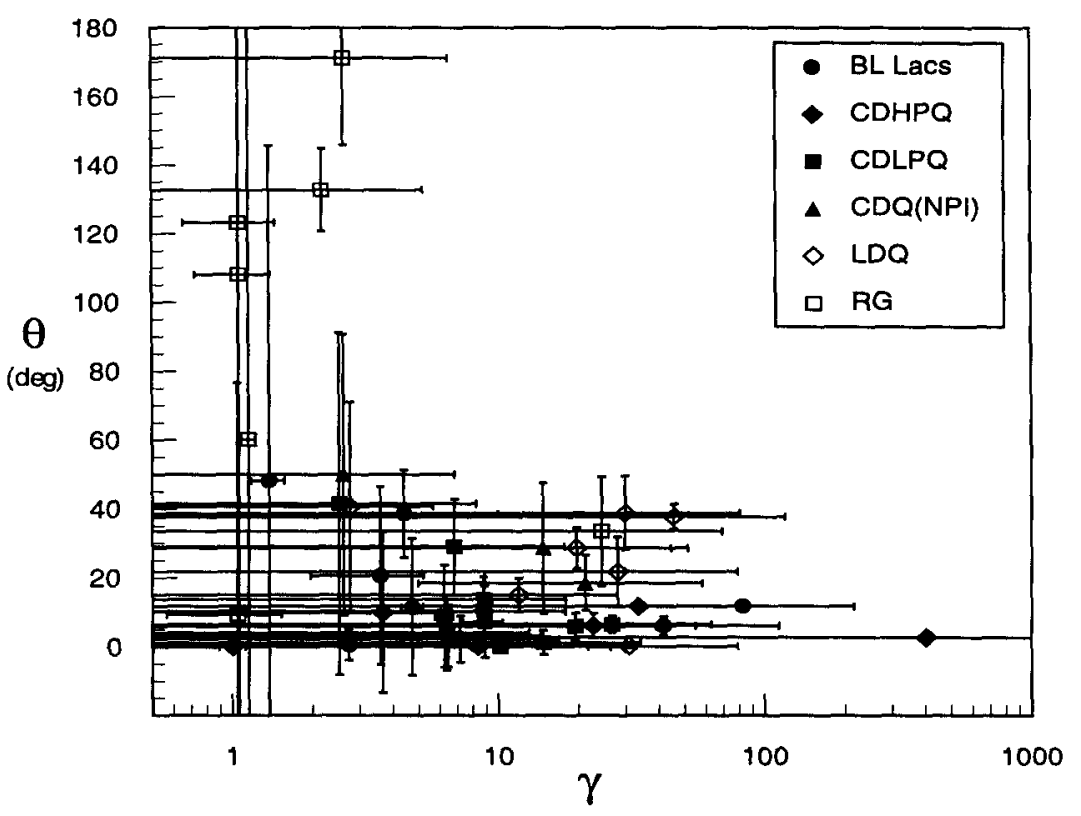

Figure 1. Estimates of $\theta$ vs. $\gamma$ using $\delta_{e q}$ and $\beta_{a p p}$.

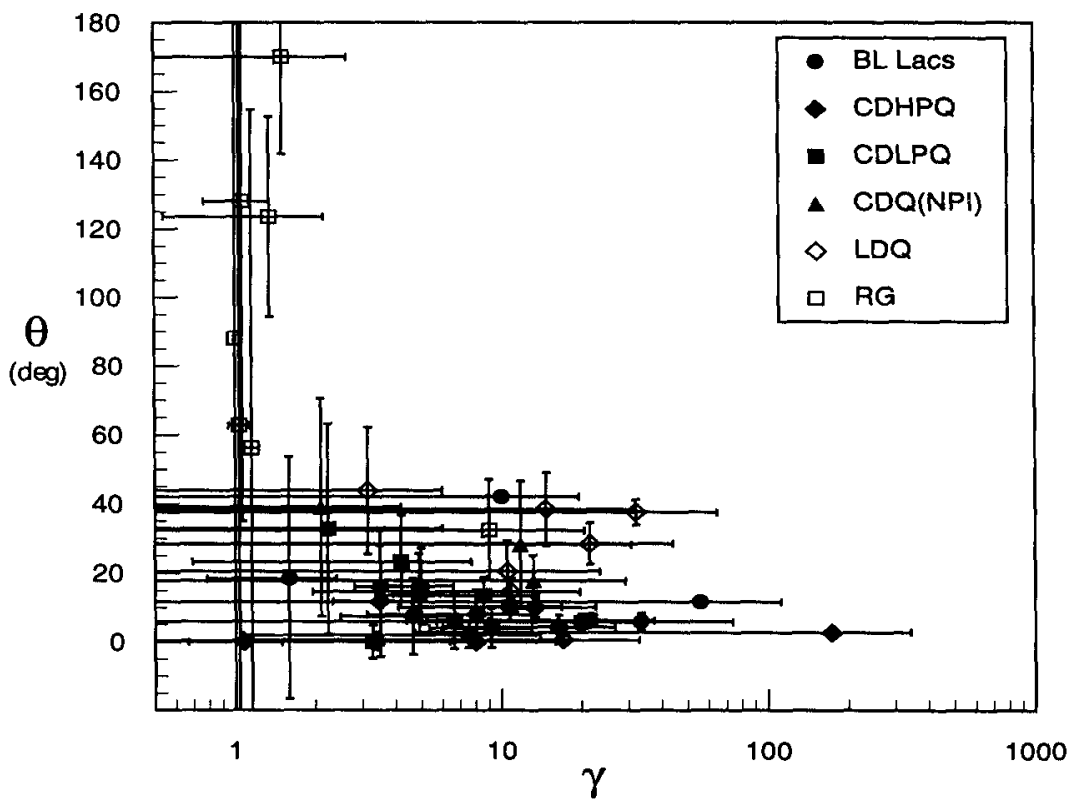

Figure 2. Estimates of $\theta$ vs. $\gamma$ using $\delta_{I C}$ and $\beta_{a p p}$. 